\title{
Albanisches Gewohnheitsrecht und Häusliche Gewalt
}

Barbara Egler

\section{Der Kanun: das albanische Gewohnheitsrecht}

Der Kanun des Lek Dukagini ist das bekannteste albanische Gewohnheitsrecht, aber es ist nicht das einzige, welches existiert. Der albanische Ausdruck für Gewohnheitsrecht ist ganz allgemein „Kanun“. Das Wort ist möglicherweise aus dem Summerischen $(\mathrm{gi}=\mathrm{Rohr})$ über das Akkadische $(\mathrm{qanu}=\mathrm{Rohr})$ ins Hebräische $(\mathrm{qane}=$ Rohr) entlehnt worden und von da aus ins Griechische (kanna $=$ Rohr) übernommen worden. Dort wurde es zu „kanon“ weitergebildet, was soviel wie „Regel, Norm“ bedeutet. Festzuhalten ist, dass das Gewohnheitsrecht der Albaner immer Ergänzungs- und zugleich Konkurrenzrecht zum staatlichen Recht war, sei es zum Recht der Türken, zum Recht des albanischen Staates nach 1912, zum Recht der Besatzungsverwaltungen im ersten und zweiten Weltkrieg, und, was den Kosovo angeht, zum Recht Jugoslawiens bzw. Serbien-Montenegros bzw. Serbiens (Elsie, Einführung S. x-xiv) bzw. Kosovos (seit 17.02.2008). Heute wird auch die Theorie vertreten, dass keine bestimmte Person Autor dieses Kanun gewesen sein soll, sondern dass er aus der logischen Konsequenz bestimmter Lebensbedingungen entstanden sei.

\section{Bestimmungen des Kanun im Zusammenhang mit häuslicher Gewalt}

Im Kanun ist beispielsweise zu lesen, dass der Herr des Hauses das Recht habe, die Leute des Hauses zu strafen, wenn sie nicht so „gehen wollen", wie es das Gedeihen des Hauses erfordert (2. Buch, 1. Kapitel, Art. 2 lit. j). Herr des Hauses oder Familienoberhaupt war gewöhnlich der Erstgeborene des Hauses oder der älteste der Brüder. Wenn dieser jedoch die für dieses Amt notwendigen Eigenschaften nicht aufwies, konnte gemäss den Regeln des Kanun auch derjenige Familienoberhaupt werden, welcher am "gescheitesten, sanftesten und sorgsamsten“" war (2. Buch, 1. Kapitel, Art. 2). Das Familienoberhaupt stand der Hausgemeinschaft vor und verwaltete sämtliches Gut. Bei gewissen Entscheidungen wie z.B. grösseren Anschaffungen oder Verkäufen (Land oder Vieh) konnte das Familienoberhaupt nicht alleine bestimmen, der Entscheid musste von allen männlichen Mitgliedern der Familie getragen werden. So wurden auch Entscheidungen im Zusammenhang mit Eheschlüssen oder Blutrache von allen Männern des Hauses gemeinsam gefällt. Das weibliche Gegenstück des männlichen $\mathrm{Fa}$ milienoberhauptes war die Frau des Hausherrn. Sie war dem Hausherrn untergeordnet, war jedoch für sämtliche Tätigkeiten des Haushaltes verantwortlich, delegierte und überwachte die Aufgaben der weiteren weiblichen Familienmitglieder. Familie oder auch Hausgemeinschaft wird gemäss Kanun die Gemeinschaft von Personen genannt, die unter einem Dach lebt. Es handelt sich dabei um die Basiseinheit der nordalbanischen Gesellschaft. Sie setzt sich aus mehreren, über die männliche Linie miteinander verwandten Kernfamilien verschiedener Generationen zusammen und konnte früher aus 60 bis 90 Personen bestehen.

Des Weiteren findet man im Kanun den Satz: „Führt sich die Frau beim Manne nicht so auf, wie es sich gehört, so gestattet der Kanun, ihr die Franse des Haares abzuschneiden und sie zu entlassen" (4. Buch, 3. Kapitel, Art. 4 Abs. 1). Auch hier wird ersichtlich, dass die Frau dem Manne zu gehorchen hat, und dass der Mann seine Frau bestrafen darf, wenn sie sich nicht so verhält, ,wie es sich gehört".

Es gibt weitere Paragraphen im Kanun, aus welchen klar hervorgeht, dass der Mann seine Frau nicht nur zurechtweisen, sondern auch gegen sie tätlich werden darf, ohne dass es irgendwelche Konsequenzen für ihn hätte: „Der Mann hat das Recht, die Frau zu tadeln und zu beraten; die Frau zu schlagen und zu binden, wenn sie seinen Anordnungen Spott bietet“ (4. Buch, 4. Kapitel, Art. 1 Abs. 1 lit. a und lit. b). Der Frau wird in dieser Situation keinerlei Schutz zugesagt, sie ist ihrem Mann ausgeliefert und kann auch ihre eigene Herkunftsfamilie nicht um Hilfe bitten, denn im Kanun steht sodann geschrieben: „Schlägt der Mann die Frau, so fällt er nach dem Gesetz nicht in Schuld, und ihre Eltern können ihn für dieses Schlagen nicht zur Rechenschaft ziehen" (4. Buch, 3. Kapitel, Art. 1 Abs. 6).

Der Mann darf seine Frau also schlagen. Die Grenze ist die Verletzung: „Verletzt der Mann die Frau und beklagt diese sich bei ihren Eltern, so wird der Mann diesen Rechenschaft geben" (4. Buch, 3. Kapitel, Art. 1 Abs. 5). Tätlichkeiten sind demnach unbeschränkt erlaubt, die Körperverletzung ist hingegen verboten und hat Konsequenzen. Was jedoch genau damit gemeint ist, dass der Mann bei den Schwiegereltern Rechenschaft ablegen muss, wird nicht definiert. Es dürfte sich um eine Art mündliche Entschuldigung handeln.

Aus dem vorher Gesagten ergibt sich, dass das Züchtigungsrecht des Ehemannes seine Grenze dort hat, wo die Ehefrau eine Körperverletzung erleidet. Die Körperverletzung ist aber nicht in jedem Fall die Richtlinie, an welche sich der Mann zu halten hat. Es gibt sogar Fälle, in denen der Mann gemäss Kanun seine Frau töten darf. „Für zwei Dinge hat die Frau die Patrone im Rücken, und für einen Grund darf ihr die Franse geschnitten und sie entlassen werden: für Untreue und für Verletzung der Freundschaft. Für diese beiden Taten der Treulosigkeit tötet der Gatte die Frau; sie bleibt ohne Schutz, ohne Gottesfrieden" (4. Buch, 3. Kapitel, Art. 4 Abs. 3). Ist die Frau dem Mann also untreu, erfährt sie keinen Schutz mehr, ihre körperliche Integrität wird nicht mehr geschützt, ja sogar ihr Recht auf Leben ist erloschen. Sie ist dem Mann vollständig ausgeliefert, und er darf sie töten.

\section{Kanun und staatliches Recht}

inerseits wird im Kanun festgehalten, dass die Ausübung von häuslicher Gewalt (bis zu einem gewissen Grad) ein Recht des Ehemannes ist, andererseits stehen diese Rechte in krassem Widerspruch zum neuen staatlichen Recht Albaniens. Die Albanische Verfassung vom 21. Oktober 1998 statuiert in Art. 122, dass alle von Albanien unterzeichneten internationalen Abkommen integrierter Bestandteil des innerstaatlichen Rechts seien und Vorrang vor nationalen Gesetzen haben (http://de.wikipedia.org/wiki/ Verfassung_Albaniens). Auch garantiert die Albanische Verfassung Rechtsgleichheit und verbietet Diskriminierung auf Grund von Geschlecht, Rasse, Religion, ethnischer Zugehörigkeit, Sprache, politischer Ansicht, etc. (Art. 18). Männer und Frauen sind, was die albanische Verfassung anbelangt, gleichberechtigt. 
Ende 2003 trat in Albanien zudem ein neues Familiengesetz in Kraft, welches spezielle Schutzregelungen für Frauen vorsieht. Die Ehefrau kann zum Beispiel verlangen, dass der Gewalt ausübende Ehemann aus der ehelichen Wohnung weggewiesen wird. Und seit dem 01.06.2007 gibt es ein Gesetz über Massnahmen gegen Gewalt in der Familie, welches bestimmt, dass die Arbeit von Polizei, Gerichten, Sozialbehörden und allfälligen weiteren Behörden koordiniert werden soll. Bei einer Meldung im Zusammenhang mit häuslicher Gewalt soll der Schutz für die Frau verbessert werden.

Auf Grund der oben aufgeführten Diskrepanz zwischen Gewohnheitsrecht einerseits und staatlichem Recht andererseits ist es nicht erstaunlich, dass die Frauen in Albanien erlebte häusliche Gewalt nicht als Delikt verstehen und somit keine Anzeige erstatten. Mindestens jede dritte Frau in Albanien erfährt im engsten Familienkreis physische Gewalt (Fleschenberg S. 1). Zudem betrachtet die Polizei vor allem in den ländlichen Gebieten Albaniens Fälle häuslicher Gewalt nicht per se als Verbrechen.

\section{Fallbeispiele}

In meiner Arbeit war ich konkret mit zwei Fällen konfrontiert, in denen häusliche Gewalt im Zusammenhang mit dem Kanun stand. In beiden Fällen hatten die Beschuldigten den Kanun nicht selbst gelesen, sondern nur davon gehört. Ich habe immer wieder die Erfahrung gemacht, dass der Inhalt einfach von Generation zu Generation mündlich weiter gegeben wird.

\subsection{Das Familienoberhaupt bestimmt}

Einer der Beschuldigten war ein aus dem Kosovo stammender Mann Mitte vierzig. Er war verheiratet und hatte drei Kinder. Bezüglich seiner Herkunft gab er an, dass die Beziehungen innerhalb seiner Familie immer gut gewesen seien. Er habe gelernt, dass die Ehefrau den Ehemann und die Kinder ihre Eltern stets zu respektieren hätten. Dies habe man denn auch im Kosovo so gelebt. Seine Kindheit sei von Respekt geprägt worden.

Dem Beschuldigten wurde vorgeworfen, mehrfach gegen seine Frau und seine Kinder tätlich geworden zu sein und seine Frau mindestens einmal mittelschwer verletzt zu haben, indem er mit einem Gegenstand auf sie eingeschlagen hatte. Zur schwerwiegenden Auseinandersetzung zwischen ihm und seiner Frau kam es, als der älteste Sohn anfing, sich für seine Mutter einzusetzen, weil diese vom Vater geschlagen wurde. Für den Beschuldigten war es unverständlich, dass sich sein Sohn auf die Seite der Mutter stellte und diese verteidigen wollte. Der Beschuldigte war grundsätzlich der Ansicht, dass ein Mann Frau und Kinder nicht schlagen dürfe, ausser wenn diese sich in seine Angelegenheiten einmischten, nämlich anfingen, selbst Entscheidungen zu treffen, obwohl dies in der Kompetenz des Mannes stünde. Auch gemäss albanischer Mentalität müssten Probleme zunächst verbal gelöst werden, erst wenn dies nichts bringe, dürfe man zur körperlichen Züchtigung schreiten.

In seiner Situation habe Reden nicht mehr geholfen. Er sah keinen Ausweg, als seine Frau und seine Kinder zu schlagen, da diese ihm nicht mehr gehorchten und sich nicht mehr an die albanischen Gewohnheiten hielten. Sie passten sich seiner Meinung nach zu stark der westeuropäischen Kultur an. Als er mit den Schlägen erst einmal angefangen habe, sei es für ihn schwierig geworden, diese wieder einzustellen. Er habe einfach immer weitermachen müssen, um das Gesicht nicht zu verlieren. Er betrachtete die Schläge als Erziehungsmassnahme. Seine Frau und seine Kinder seien mit dieser Mentalität immer vertraut gewesen, sie hätten diese bis zu einem gewissen Grad akzeptiert. Für ihn wurde es schwierig, die Grenze zu ziehen zwischen erlaubtem Züchtigungsrecht und strafbarer Handlung. Ihm habe nie jemand erklärt, was eigentlich erlaubt sei und was nicht. Dass er seine Frau und seine Kinder gar nicht schlagen dürfe, habe er nicht gewusst. Seine Motivation, Frau und Kinder zu schlagen, hatte darin bestanden, seine Familie „vor Schwierigkeiten“ zu bewahren, sein oberstes Ziel war der Schutz seiner Familie, damit diese nicht von den guten, alten Traditionen abkamen. Als die gewöhnlichen Schläge nicht mehr halfen, griff er zu einem Gegenstand und schlug damit auf seine Frau ein, dabei verlor er die Kontrolle und verletzte seine Frau dabei mittelschwer.

Die Ehefrau des Beschuldigten gab in den Vernehmungen an, seit der Hochzeit mit ihrem Mann regelmässig von diesem geschlagen, in der Regel aber nicht verletzt worden zu sein. Aber nicht nur ihr Ehemann sondern auch ihr Schwager habe sie geschlagen. Der älteste Bruder ihres Ehemannes habe innerhalb der Familie ihres Mannes die Rolle des Familienoberhauptes eingenommen, er habe über die wichtigen Angelegenheiten bestimmt, welche sämtliche Kernfamilien in und ausserhalb des Kosovo betroffen hät- ten. Die Kernfamilie des Beschuldigten (er, seine Frau und seine Kinder) waren demnach nicht autonom, sondern hatten sich regelmässig an die Anordnungen des ältesten Bruders des Beschuldigen zu halten, wie dies gemäss Kanun vorgeschrieben ist.

Als sie von ihrem Ehemann mit einem Gegenstand verletzt wurde, verbot er ihr, einen Arzt aufzusuchen. Auch das Familienoberhaupt mischte sich in die Angelegenheit ein und schlug vor, dass der Fall innerhalb der Familie gelöst und die Polizei nicht kontaktiert werde. Sie hatte grosse Angst vor ihrem Ehemann und vor dessen Bruder, ihre Herkunftsfamilie konnte ihr in dieser Situation nicht helfen. Sie gab an, auch regelmässig von ihrem Ehemann mit dem Tode bedroht worden zu sein, diese Drohungen seien alltäglich gewesen und sie habe sie daher nicht ernst genommen. Als aber ihr Mann auf sie eingeschlagen, und während des Schlagens die Kontrolle verloren und nicht mehr aufgehört habe, dachte sie, dass er jetzt seine Drohungen in die Tat umsetzen und sie tatsächlich umbringen werde.

Der erwachsene Sohn des Beschuldigten gab in den Vernehmungen an, dass er die Polizei gerufen habe, als seine Mutter von seinem Vater verletzt wurde. In dieser einen Situation habe der Vater die Kontrolle definitiv verloren, und die Mutter spitalreif geschlagen. Früher sei der Vater einfach nur tätlich gewesen, ohne dabei jemanden zu verletzen. Das habe man in der Familie hingenommen, ohne sich gegen den Vater zu wehren. Alle Familienmitglieder seien in regelmässigen Abständen geschlagen worden, wenn sie sich nicht gemäss den Anordnungen des Vaters verhalten hätten. Der Sohn gab an, dass das eigentliche Problem nicht der Vater sei, sondern der älteste Bruder des Vaters, welcher in der Familie das eigentliche Sagen habe. Das Familienoberhaupt liess nicht zu, dass er sich seine eigenen Freunde suchte und selbst bestimmte, mit welcher Frau er zusammen sein wollte. Das Familienoberhaupt gab auch Anweisungen betreffend Züchtigung von Frau und Kindern. Die Kernfamilie war nicht autonom, sondern lebte stets nach den Bestimmungen des $\mathrm{Fa}$ milienoberhauptes. Auch das Geld, welches die einzelnen Familienmitglieder verdienten, wurde nach den Vorschriften des Familienoberhauptes angelegt und investiert. Niemand konnte über sein eigenes Leben verfügen oder seinen eigenen Besitz verwalten, alles wurde vom ältesten Bruder des Vaters angeordnet. Wenn die Mutter oder eines der Kinder sich nun zum Beispiel in eine finan- 
zielle Angelegenheit einmischte, dann konnte es sein, dass sowohl der Vater als auch das Familienoberhaupt diesen Ungehorsam mit Schlägen bestraften. Niemand durfte sich den Anweisungen des Familienoberhauptes und seiner Brüder widersetzen. Der Sohn berichtete, auch er sei regelmässig vom Onkel geschlagen worden, wenn er sich getraut habe, etwas gegen die Regeln innerhalb der Familie zu sagen.

\subsection{Wenn die Frau den Mann ver-}

lässt, ist seine Ehre verletzt

Beim zweiten Beschuldigten handelte es sich um einen dreissigjährigen Mann, ebenfalls aus dem Kosovo stammend. Er war verheiratet und hatte zwei Kinder. Dem Beschuldigten wurde vorgeworfen, mehrfach seine Frau mit dem Tode bedroht zu haben, nachdem diese ihn wegen eines anderen Mannes verlassen hatte. Der Beschuldigte bestritt während des Ermittlungsverfahrens sämtliche Vorwürfe, betonte aber immer wieder, dass er wisse, dass seine Frau einen neuen Freund habe, und dass es für diesen Fall nach seinem Brauchtum klare Vorschriften gebe. Es sei an dieser Stelle nochmals auf das
4. Buch, 3. Kapitel, Art. 4 Abs. 3 verwiesen: „Für zwei Dinge hat die Frau die Patrone im Rücken, und für einen Grund darf ihr die Franse geschnitten und sie entlassen werden: für Untreue und für Verletzung der Freundschaft. Für diese beiden Taten der Treulosigkeit tötet der Gatte die Frau; sie bleibt ohne Schutz, ohne Gottesfrieden “. Wird eine Frau untreu, kann der Mann sie töten.

Der Beschuldigte in unserem Fall kannte diese Bestimmung ohne Zweifel. Er betonte jedoch immer wieder, dass er diese Bestimmung nicht vollziehen werde. Er kenne den Kanun zwar, aber er wolle schliesslich nicht, dass seine Kinder ohne Mutter aufwachsen müssten. Aber seine Frau müsse wissen, dass in einem solchen Fall eine „Reaktion" erfolgen könne. Sie habe ihn betrogen, und er könne für nichts garantieren. Seine Brüder seien schliesslich auch noch da, und diese seien ganz besonders auf die Situation in seiner Ehe aufmerksam geworden und könnten eine Trennung unter keinen Umständen dulden, erst recht nicht wenn der Grund dafür die Untreue der Ehefrau sei. Er habe denn seiner Frau auch nie gedroht, sondern sie immer nur auf die Folgen ge- mäss Kanun aufmerksam gemacht und sie darauf hingewiesen, dass sie sich an das alte Brauchtum im Kosovo erinnern solle. Er habe seine Frau daher nicht bedroht, sondern nur "gewarnt“.

Unter anderem wurde dem Beschuldigten auch vorgeworfen, seiner Frau einen Zeitungsartikel geschickt zu haben, in welchem ein Fall eines Ehrenmordes geschildert wurde: Ein Mann hatte seine Exfrau nur wenige Stunden nach der Scheidung niedergestochen.

Barbara Egeler Dormann ist Untersuchungsrichterin in der Schweiz, Rheinstrasse 12, CH-4410 Liestal

\section{Literaturverzeichnis:}

- Ahmeti Zef (2006). Das Strafrecht im Kanun von Lekë Dukagjini. St. Gallen.

- Elsie Robert (2001). Der Kanun - Das albanische Gewohnheitsrecht nach dem so genannten Kanun des Lekë Dukagiini, kodifiziert von Shtjefën Gjeçovi, ins Deutsche übersetzt von Marie Amélie Freiin von Godin. Olzheim/Eifel.

- Fleschenberg Jana (2005). Familiäre Gewalt in Albanien, Hintergrundinformationen. Amnesty International. Aufsatz im Internet.

\section{Fremdenfeindliche Straftaten und ihre statistische Erfassung - Eine Zwischenbilanz}

\section{Einleitung}

Kriminalität ist definitionsabhängig und wird unter anderem von den Instanzen sozialer Kontrolle konstruiert. Die Begrifflichkeiten und Definitionen, die der Erfassung und Abbildung krimineller Erscheinungsformen zugrunde gelegt werden, sind ebenso wie die Phänomene selbst zeitlichen Veränderungsprozessen und politischen Strömungen unterworfen. Von offizieller Seite besteht das Bestreben, auf neue Ausprägungen bestimmter Delikte dynamisch zu reagieren. Dies geschieht einerseits durch Akte der Gesetzgebung, was nicht selten mit Strafschärfungen einhergeht. ${ }^{1}$ Zum anderen wird versucht, über Veränderungen der statistischen Erfassung krimineller Verhaltens-weisen eine Anpassung an gewandelte Realitäten vorzunehmen. Letzteres geschah bekanntlich auch in Bezug auf fremdenfeindliche Straftaten. Während diese bis in die 1980er Jahre hinein kaum Beachtung fanden und nicht als besonderes Problem wahrgenommen wurden, gelangten sie im Zuge der Asyldebatte zu Beginn der 1990er Jahre verstärkt ins Blickfeld der Öffentlichkeit. ${ }^{2}$ In dieser Zeit kam es zu zahlreichen Brandanschlägen und Gewaltdelikten (etwa in Solingen, Rostock und Mölln), die großes öffentliches Aufsehen erregten und ferner dazu führten, dass sich die Wissenschaft verstärkt den Hintergründen und Entstehungsbedingungen dieser Taten zuwendete. Kontrollinstanzen, Medien und Politiker sahen sich mit dem Kriminalitätsphänomen „Fremden-feindlichkeit“ konfrontiert, das beobachtet werden musste und deshalb einer statistischen Erfassung bedurfte.

\section{Die Situation bis zum 31.12.2000}

Für die Registrierung von Straftaten der vorgenannten Art stand zunächst die „Po-

\section{Sebastian Presse und Mario Bachmann}

lizeiliche Kriminalstatistik für Staatsschutzdelikte (PKS-S)“ zur Verfügung. Hier erfasste das Bundes-kriminalamt als Teil der Staatsschutzdelikte rechts- und linksextremistische Straftaten. Unter dem zentralen Begriff „Extremismus“ wurden dabei solche Bestrebungen verstanden, die sich gegen den Kernbestand der freiheitlich demokratischen Grundordnung richteten und diese zu überwinden suchten. ${ }^{3}$ Fremdenfeindlichkeit als gesellschaftlicher Hintergrund von Straftaten wurde allerdings nicht eigens ausgewiesen. ${ }^{4}$ Dies geschah erst ab März 1992 in der KPMD-S („Kriminalpoli-zeilicher Meldedienst in Staatsschutzsachen "). Nach der vom Bundeskriminalamt und der von den Länderpolizeien entwickelten Definition handelte es sich bei fremdenfeindlichen Straftaten um solche, die ,in der Zielrichtung gegen Personen begangen werden, denen die Täter aus intoleranter Haltung heraus aufgrund ibrer Nationalität, Ras- 